\title{
Delayed diagnosis of a primary intraosseous squamous cell carcinoma: A case report
}

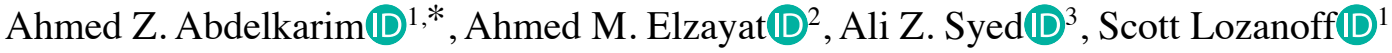 \\ ${ }^{1}$ Department of Anatomy, Biochemistry and Physiology, University of Hawai'i School of Medicine, Honolulu, HI, USA \\ ${ }^{2}$ Department of Oral and Maxillofacial Surgery, Insurance Hospital, Suez, Egypt \\ ${ }^{3}$ Oral and Maxillofacial Medicine and Diagnostics Science, CWRU School of Dental Medicine, Cleveland, OH, USA
}

\section{ABSTRACT}

Primary intraosseous squamous cell carcinoma is a rare malignant central jaw tumor derived from odontogenic epithelial remnants. Predominantly, it affects mandible, although both jaw bones may be involved. This report describes a 60 -yearold man who was initially misdiagnosed with a periapical infection related to the right lower wisdom tooth. After four months, the patient presented to a private dental clinic with a massive swelling at the right side of the mandible. Panoramic radiographs and advanced imaging revealed a lesion with complete erosion of the right ramus, which extended to the orbital floor. A biopsy from the mandibular angle revealed large pleomorphic atypical squamous cells, which is the primary microscopic feature of a poorly differentiated squamous cell carcinoma.(Imaging Sci Dent 2019; 49: 71-1)

KEY WORDS: Carcinoma, Squamous Cell; Mandible; Delayed Diagnosis; Computed Tomography, X-Ray; Magnetic Resonance Imaging

Primary intraosseous squamous cell carcinoma (PIOSCC) is an uncommon neoplasm, defined as a squamous cell carcinoma (SCC) that develops within the jaw bones and arises from remnants of odontogenic epithelium with no initial connection to the oral mucosa. ${ }^{1}$ In 2005 , the World Health Organization (WHO) divided PIOSCC into 3 types: solid-type carcinoma, carcinoma arising from a keratocystic odontogenic tumor, and carcinoma arising from an odontogenic cyst. ${ }^{2}$ The definitive diagnosis of PIOSCC is often problematic, and the diagnostic criteria for PIOSCC still remains elusive. Some studies have reported that one of the histopathological definitions of PIOSCC is a SCC arising within the jawbone that does not involve the oral mucosa. ${ }^{3,4}$

PIOSCC is estimated to account for $12 \%$ of all cases of oral cancer. ${ }^{1}$ The majority of PIOSCCs arise from odontogenic cysts, including dentigerous cysts and keratocystic odontogenic tumors, and only rarely do they originate from residual periapical cysts. ${ }^{5}$ Commonly reported clinical features of PIOSCC include jaw swelling, pain, and sensory

Received August 26, 2018; Revised October 18, 2018; Accepted November 18, 2018 *Correspondence to: Dr. Ahmed Z. Abdelkarim

Department of Anatomy, Biochemistry and Physiology, University of Hawai'i School of Medicine, Honolulu, HI 96813, USA

Tel) 1-808-725-9954, E-mail) ahmedz@hawaii.edu disturbances. ${ }^{4}$ Before the diagnosis of PIOSCC, the existence of a primary tumor in another site must be ruled out. ${ }^{4}$ Histological findings are often not pathognomonic for the diagnosis of PIOSCC. ${ }^{5}$

The radiographic features of PIOSCC show considerable variation both within and between each type. ${ }^{6}$ Solid-type PIOSCC commonly displays an osteolytic appearance with ill-defined irregular margins, whereas the other types are often indistinguishable from benign jawbone lesions with well-defined margins during the initial phase. ${ }^{2}$ Larger PIOSCCs of all kinds have various destructive effects on the jawbone. ${ }^{2}$

The purpose of this article is to present a case of PIO SCC, along with a review of the clinical, radiological, and microscopic characteristics of PIOSCC that led to a delayed diagnosis. This case adds to the very few instances of definitive PIOSCC diagnosis described in the literature.

\section{Case Report}

In March 2018, a 60-year-old man presented to a private dental clinic with a history of painful swelling in the right angle of the mandible. The patient reported that in December 2017, he had visited another local dental clinic with a chief 

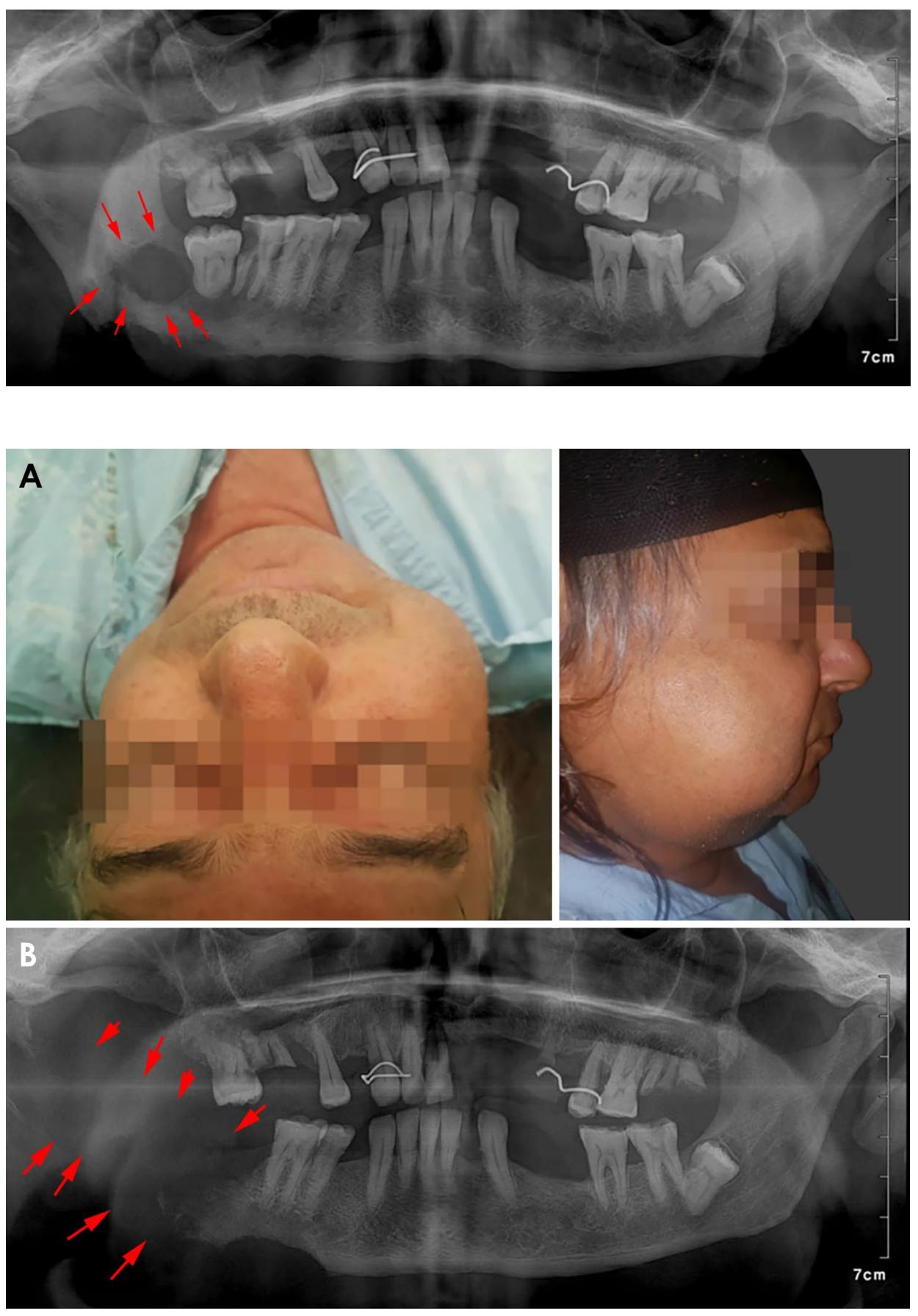

Fig. 1. Panoramic radiograph shows an ill-defined radiolucent lesion posterior to the right third molar with erosion of the inferior border of the mandible.

Fig. 2. Clinical picture over extraoral examination and panoramic radiograph. A. Supine and lateral views show a large swelling at the right side of the face. B. Panoramic radiograph shows complete erosion of the right ramus, condyle, and a portion of the mandibular body. complaint related to pain in the right posterior mandibular region. His past medical history included controlled type 2 diabetes. The dentist requested a panoramic radiograph, and the diagnosis was a periapical infection related to the right third molar and badly decayed right second molar, and an extraction was performed based on the patient's history. After extraction, the patient reported continuous pain with an unhealed socket, and an antibiotic regime was initiated. As a result of the unresolved pain, and without a prescription from his physician, he packed the unhealed wound with di- clofenac sodium daily.

A panoramic radiograph was acquired from his first visit (Fig. 1), and it revealed an ill-defined radiolucent lesion posterior to the right third molar, as well as an abnormally irregular inferior border of the mandible. The clinical examination record did not mention any observation of an intraoral soft tissue mass or ulceration at that time. An extraoral examination revealed a swelling with paresthesia in the right mandibular angle (Fig. 2A). A panoramic radiograph was requested and showed complete erosion of the right ra- 
mus, with the lesion extending to the body of the mandible (Fig. 2B). The radiographic signs were highly suggestive of aggressive neoplastic changes, and advanced imaging was therefore recommended. Multi-slice computed tomography without contrast revealed a large soft-tissue mass lesion occupying the right side of the parapharyngeal region, extending from the inferior orbital margin to the lower neck region, resulting in a prominent right anterolateral contour bulge (Fig. 3). The lesion had infiltrated the masseter muscle. A complete absence of the right mandibular ramus and temporomandibular joint (TMJ) was observed, as well as erosion of the distal mandibular body. The right maxillary sinus showed erosion of its posterolateral boundary, mild mucosal thickening, and a large cyst with a calcific margin and fluid content. The left TMJ was intact, and no osteoarthritic changes were noticed. These imaging features suggested a large, osteolytic, neoplastic lesion.

Magnetic resonance imaging (MRI) was performed in the head and neck region to reveal the soft-tissue extent of the lesion mass (Fig. 4). A large irregular heterogenous soft tissue mass involving the right hemi-mandible was observed, including the condylar and coronoid processes and the mandibular ramus, and most of the mandibular body had been replaced by a broad, ill-defined, soft-tissue mass lesion measuring $8.8 \times 4.5 \times 5 \mathrm{~cm}$. The lesion was seen extending superiorly into the mandibular fossa of the temporal bone, inferiorly to the level of the C3 vertebra, and medially involving the medial and lateral pterygoid muscles. Laterally, the lesion had invaded the masseter muscle and extended into the right subcutaneous facial fat planes, with an indistinct line of cleavage from the parotid gland. Posteriorly, it was seen to be intimately related to the right maxillary artery. The mass displayed a low signal in T1 imaging, and an intermediate signal in T2 imaging, with hyperintense foci. In post-contrast $\mathrm{T} 1$ imaging, the lesion showed intense post-contrast enhancement and was seen to have infiltrated the adjacent muscles.

Another well-defined rounded lesion, measuring $2 \times$ $2.2 \times 2.3 \mathrm{~cm}$ with a similar imaging pattern, was observed inferior to the neoplasm. It was closely related to the right submandibular gland, with a vague line of cleavage. In the right maxillary sinus, a soft-tissue mass (predominantly low-signal in T1 imaging and high-signal with a low-signal area in the center in T2 imaging) was seen, measuring $2 \times 2.5 \mathrm{~cm}$, along with an obliterated osteomeatal complex and invasion of the inferomedial maxillary wall with significant post-contrast enhancement (Fig. 4C). These MRI features of the maxillary lesion indicated that the content

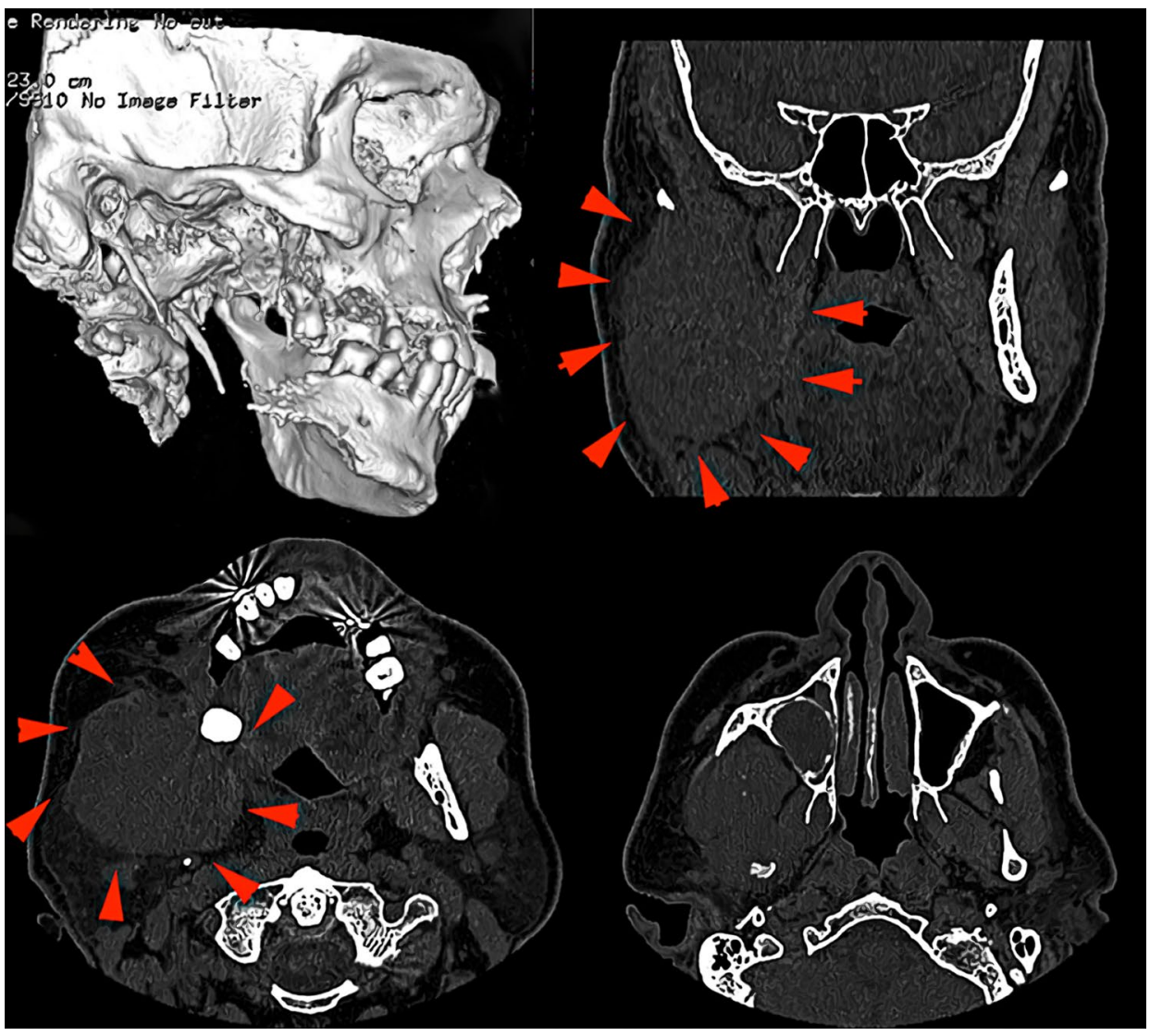

Fig. 3. Multi-slice computed tomography. A 3-dimensional reconstruction image shows complete erosion of the ramus. Axial, coronal, and sagittal slices show extension of the lesion (red arrows). 

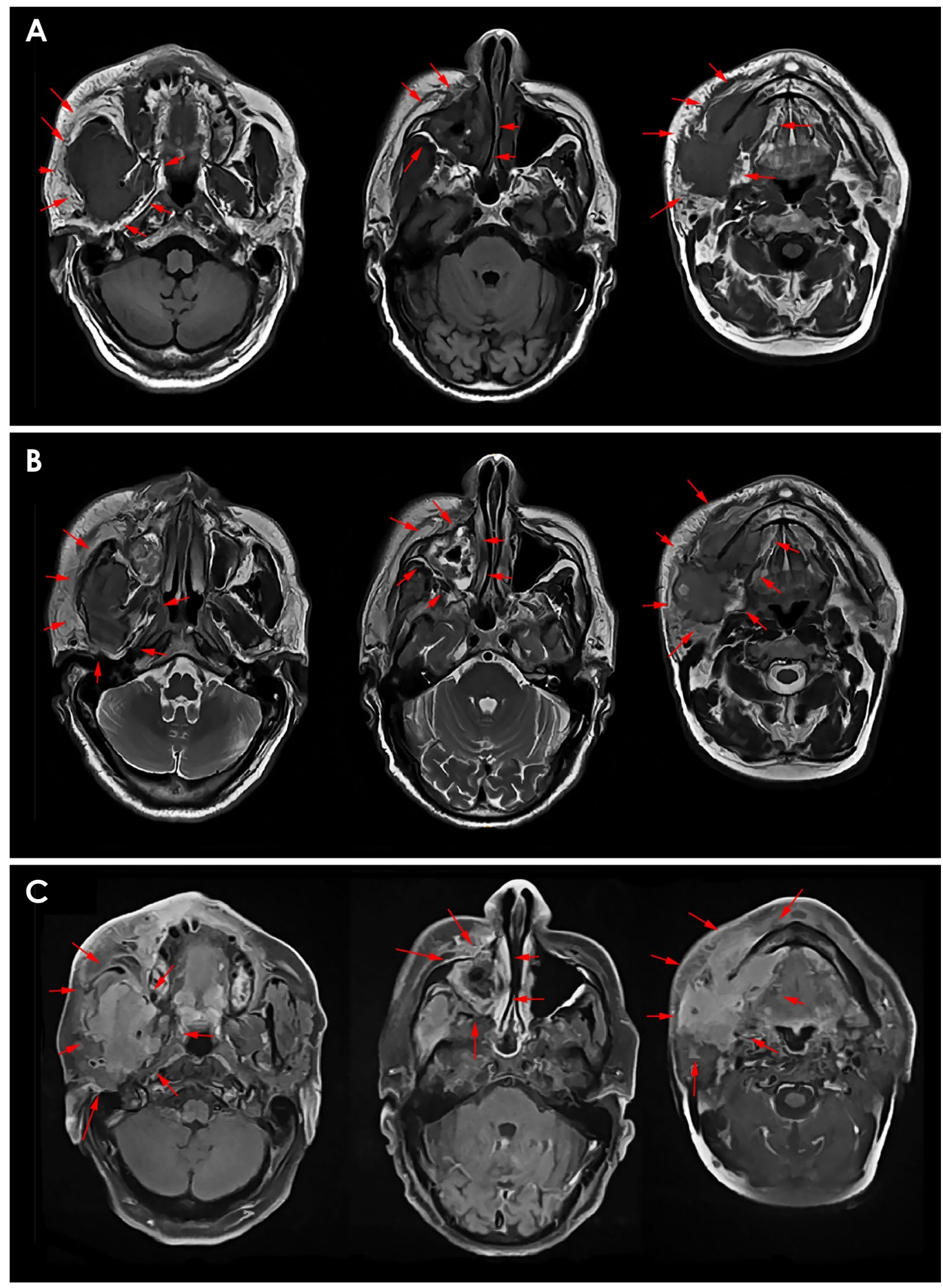

Fig. 4. Magnetic resonance imaging examination. Series of axial T1 (A), T2 (B), and post-contrast axial T1 (C) images showing the extension of the lesion (arrows) in the pterygoid region, maxillary sinus, and submandibular area (columns from left to right).

was an inflammatory process not consistent with the main lesion. This excluded the possibility that the main lesion extended to the sinus, and the lesion in that area was diagnosed as sinusitis. Multiple bilateral enhancing submandibular and upper cervical enlarged lymph nodes were noticed, with the most significant measuring $1.4 \mathrm{~cm}$ on the right side. The differential diagnoses were odontogenic carcinoma, metastatic carcinoma, and sarcoma.

Two specimens were taken for histopathological examination (Fig. 5). The first specimen was obtained from 

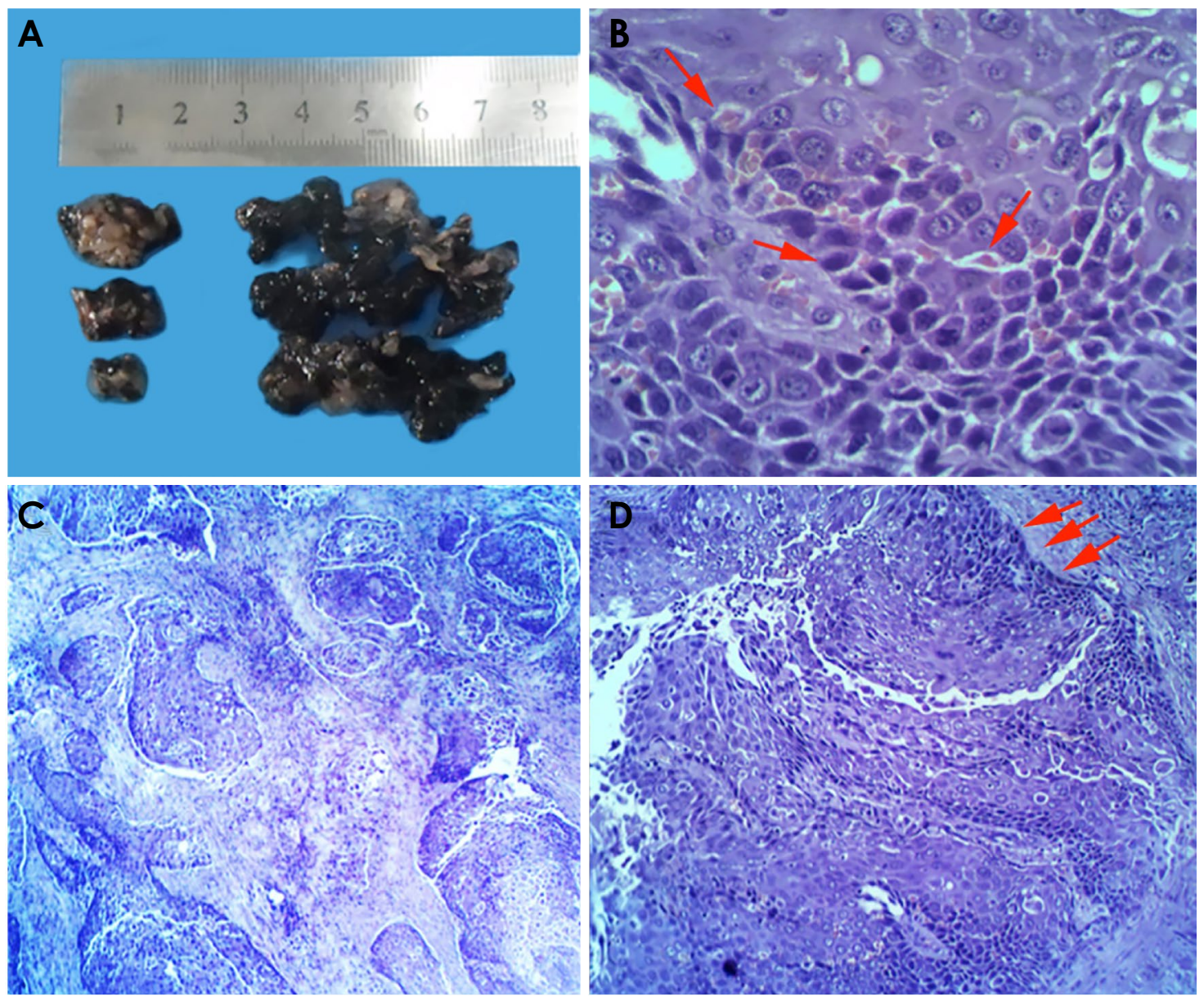

Fig. 5. Gross biopsy and histopathology examination. A. Two specimens from the angle of the mandible ( 3 pieces, left) and the maxillary sinus (multiple pieces, right). B. Section shows groups of large pleomorphic atypical squamous cells (arrows) with a high nucleoplasm ratio and focal intracytoplasmic keratin formation (H\&E stain, $\times 400)$. C. Numerous irregularly shaped solid epithelial islands of varying sizes in the connective tissue $(\mathrm{H} \& \mathrm{E}$ stain, $\times 40)$. D. The basal cells are arranged in a plexiform pattern with palisading of the peripheral cells $(\mathrm{H} \& \mathrm{E}$ stain, $\times 100)$ (red arrows).

the soft tissue mass around the angle of the mandible by an extraoral approach. The second sample was obtained from the maxillary sinus by enucleation of the cyst using Caldwell-Luc antrostomy (Fig. 5A). For the first specimen, the microscopic examination revealed fibro-fatty tissue infiltrated by a tumor mass of large pleomorphic atypical squamous cells with a high nucleoplasm ratio and focal intracytoplasmic keratin formation (Fig. 5B). The intervening stroma was heavily infiltrated by mixed inflammatory cells with numerous mitotic figures. Histopathological examination of hematoxylin and eosin-stained slides showed plentiful irregular-shaped solid epithelial islands of differing sizes in the connective tissue (Fig. 5C). The basal cells vwere arranged in a plexiform pattern with palisading of the peripheral cells (Fig. 5D). Microscopic examination of the maxillary sinus specimen revealed soft tissue infiltrated by lymphocytes, plasma cells, and fragments of necrotic bony spicules. Based on the microscopic findings, a final diagnosis of solid-type PIOSCC was made.

\section{Discussion}

In this case report, the authors present a very rare case of PIOSCC. Diagnosis of PIOSCC may be difficult, as it must be differentiated from carcinoma arising from the epithelial lining of the maxillary sinus, from carcinoma originating from the alveolar mucosa epithelium that has invaded the bone, and from tumor metastasis to the jaw. Due to its rarity, its incidence, prevalence, and etiology are still not fully understood. ${ }^{7}$

PIOSCC is a rare malignant odontogenic tumor that accounts for approximately $1 \%-2.5 \%$ of all odontogenic 
tumors. ${ }^{8}$ PIOSCC displays a predilection for males $(2: 1$ male-to-female ratio). ${ }^{2}$ Although it can occur at any age, it most commonly occurs in the fifth decade of life. ${ }^{1}$ PIOSCC is more often found in the mandibular body and posterior mandible than in the maxilla. ${ }^{1}$ In our case, a 60 -year-old male patient was affected, and the lesion in the present case involved the masticator space and the infra-temporal region up to the level of the cranial base.

These findings are similar to the data published by Huang et al., ${ }^{9}$ who studied a total of 39 PIOSCCs (solid type) at Peking University School and Hospital of Stomatology (Beijing, China) from 1985 to 2006. Their findings are in concordance with those of Thomas et al. ${ }^{10}$ The etiology of PIOSCC is unclear, but the most common factor may be a reactive inflammatory stimulus with or without a predisposing genetic cofactor. ${ }^{10}$

Clinically, PIOSCC is associated with various symptoms depending on its location, size, and type. These symptoms include pain, swelling, sensory disturbances, and routine dental disorders. ${ }^{11}$ The 60 -year-old male patient described herein experienced a tingling sensation in the lower left gingiva of his molar region with paresthesia of the lower lip.

The radiological and clinical characteristics of PIOSCCs are similar to those of odontogenic tumors. In some instances, early-stage PIOSCC may mimic routine dental disorders, such as periapical and periodontal disease, which may lead to misdiagnosis or delayed diagnosis. ${ }^{12}$ In our case, the patient's first dentist misdiagnosed the lesion, in its early stage, as an inflammatory odontogenic condition. Radiographically, in most cases, osteolytic bone changes are characterized by poorly defined, diffuse, and irregular margins. ${ }^{13}$ As presented in Figure 1, the lesion showed similar features, with erosion at the inferior mandibular border.

MRI has advantages over other imaging techniques for the anatomical assessment of head and neck cancers, especially with regard to mapping the primary tumor extent. ${ }^{14}$ For this patient, the primary lesion involved the masticator space with extension to the infratemporal fossa and adjacent muscles. MRI ruled out the extension of the lesion to the maxillary sinus.

The definitive diagnosis of PIOSCC is often challenging, since malignant tumors that have metastasized to the jaw from distant sites, tumors originating from the maxillary sinus, and alveolar carcinomas that have invaded the bone from the surface must be ruled out. ${ }^{15}$ In the present case, the differential diagnoses were odontogenic carcinoma, metastatic carcinoma, or sarcoma. This differential diagnosis was formulated because the radiographic lesion was destructive, with ill-defined and ragged borders. The possi- bility of a metastatic tumor was excluded since the patient had no history of a primary tumor elsewhere in the body. Osteosarcoma and chondrosarcoma were considered. Chondrosarcomas are rarely found in the jaws, and generally occur in the anterior alveolar process of the maxilla, the alveolar ridge of the premolar-molar region, and the angle of the mandible. However, they are painless and slow-growing in their early stages. Osteosarcomas are rarely seen in the jaw bones, which account for only $7 \%$ of all cases, but are more frequently seen in long bones. Moreover, osteosarcomas generally show a moth-eaten appearance due their rapid growth pattern in the initial osteolytic stage. The age of occurrence is mainly during the third decade of life. In the case described herein, the radiographic findings did not show a sunray appearance and Codman's triangle, which are commonly seen in cases of osteosarcoma. ${ }^{7}$

PIOSCC, which develops from remnants of the odontogenic epithelium, is defined as an SCC arising within the jawbones. In 1972, the WHO suggested the term primary intraosseous odontogenic carcinoma (PIOC). ${ }^{11}$ Subsequently, the WHO classification was modified by adding ameloblastic carcinoma. ${ }^{12}$ The classification was further revised in $1984,{ }^{16}$ and intraosseous mucoepidermoid carcinoma was added to the classification of PIOC in $1989 .{ }^{17}$ In 2005, the WHO established the term PIOSCC and categorized it into 3 types: solid-type carcinoma, carcinoma arising from a keratocystic odontogenic tumor, and carcinoma arising from an odontogenic cyst. ${ }^{2}$ The detailed subcategories of PIOSCC reflect 3 characteristic etiological processes. PIOSCC can arise as a solid tumor invades the marrow space and induces osseous resorption, as an SCC originating from the lining of an odontogenic cyst, or as an SCC associated with other benign epithelial odontogenic tumors. ${ }^{2,3}$ In the present case, a histopathological examination revealed that a tumor mass was growing in the bone, and no finding was indicative of direct invasion of the SCC from the covering squamous epithelium. This conclusion was also supported by the clinical history of the patient's first visit. The tumor cells displayed the characteristic features of SCC. No signs were found of another odontogenic cyst or tumor, or of any other primary SCC.

In conclusion, the present case highlights the importance of a thorough interpretation of radiographs, as failure to read radiographs thoroughly could potentially delay the diagnosis or lead to a misdiagnosis, as happened in this case.

\section{References}

1. Bodner L, Manor E, Shear M, van der Waal I. Primary intraosseous squamous cell carcinoma arising in an odontogenic cyst: a clinicopathologic analysis of 116 reported cases. J Oral Pathol 
Med 2011; 40: 733-8.

2. Williams MD. Update from the 4th Edition of the World Health Organization Classification of Head and Neck Tumours: Mucosal Melanomas. Head Neck Pathol 2017; 11: 110-7.

3. Matsuzaki H, Katase N, Matsumura T, Hara M, Yanagi Y, Nagatsuka $\mathrm{H}$, et al. Solid-type primary intraosseous squamous cell carcinoma of the mandible: a case report with histopathological and imaging features. Oral Surg Oral Med Oral Pathol Oral Radiol 2012; 114: e71-7.

4. Suei Y, Tanimoto K, Taguchi A, Wada T. Primary intraosseous carcinoma: review of the literature and diagnostic criteria. J Oral Maxillofac Surg 1994; 52: 580-3.

5. Woolgar JA, Triantafyllou A, Ferlito A, Devaney KO, Lewis JS Jr, Rinaldo A, et al. Intraosseous carcinoma of the jaws: a clinicopathologic review. Part II: odontogenic carcinomas. Head Neck 2013; 35: 902-5.

6. Sengupta S, Vij H, Vij R. Primary intraosseous carcinoma of the mandible: a report of two cases. J Oral Maxillofac Pathol 2010; 14: 69-72.

7. Thakur A, Tupkari JV, Joy T, Gogri AA. Primary intraosseous squamous cell carcinoma-a rare odontogenic malignancy. $\mathrm{J}$ Oral Maxillofac Pathol 2017; 21: 320.

8. Tawfik MA, Zyada MM. Odontogenic tumors in Dakahlia, Egypt: analysis of 82 cases. Oral Surg Oral Med Oral Pathol Oral Radiol Endod 2010; 109: e67-73.

9. Huang JW, Luo HY, Li Q, Li TJ. Primary intraosseous squamous cell carcinoma of the jaws. Clinicopathologic presentation and prognostic factors. Arch Pathol Lab Med 2009; 133: 1834-40.

10. Thomas G, Pandey M, Mathew A, Abraham EK, Francis A, Somanathan T, et al. Primary intraosseous carcinoma of the jaw: pooled analysis of world literature and report of two new cases. Int J Oral Maxillofac Surg 2001; 30: 349-55.

11. Suei Y, Taguchi A, Tanimoto K. Recommendation of modified classification for odontogenic carcinomas. Med Hypotheses 2004; 62: 382-6.

12. Elzay RP. Primary intraosseous carcinoma of the jaws. Review and update of odontogenic carcinomas. Oral Surg Oral Med Oral Pathol 1982; 54: 299-303.

13. Reichart P, Philipsen HP. Odontogenic tumors and allied lesions. London: Quintessence Pub; 2004. p. 205-25.

14. Dai YL, King AD. State of the art MRI in head and neck cancer. Clin Radiol 2018; 73: 45-59.

15. Grisar K, Schol M, Hauben E, Schoenaers J, Politis C. Primary intraosseous squamous cell carcinoma of the mandible arising from an infected odontogenic cyst: a case report and review of the literature. Oncol Lett 2016; 12: 5327-31.

16. Slootweg PJ, Müller H. Malignant ameloblastoma or ameloblastic carcinoma. Oral Surg Oral Med Oral Pathol 1984; 57:168-76.

17. Waldron CA, Mustoe TA. Primary intraosseous carcinoma of the mandible with probable origin in an odontogenic cyst. Oral Surg Oral Med Oral Pathol 1989; 67: 716-24. 